\title{
Histopathological lesions compatible with nymphs of Linguatula serrata in bovine liver
}

\author{
Pamela Morales Muñoz ${ }^{\mathrm{a}^{*}}$, Miguel Carrillo Parraguez ${ }^{\mathrm{a}, \mathrm{b}}$, \\ María González Marambio ${ }^{\mathrm{a}}$, Francisco Carvallo Chaigneau ${ }^{c}$
}

\begin{abstract}
Linguatula serrata is the causative parasite of Linguatulosis, a disease that not only produces economic losses in cattle but also represents a public health risk due to its zoonotic nature. This study aimed to microscopically characterise the compatible lesions produced by this parasite in bovine liver collected at a slaughterhouse in the city of Curicó, Chile. Histologic compatible lesions with Linguatulosis were observed in 18 out of 269 livers. Furthermore, nymphs were visualised in 3 cases, allowing an etiologic diagnosis. Microscopic lesions containing nymphs demonstrated two patterns of inflammatory reactions, one pattern with a predominance of eosinophils, accompanied by lymphocytes and some macrophages, and another pattern with a predominance of lymphocytes and plasma cells with some macrophages. A microscopic characterisation was performed in compatible lesions without nymphs ( $\mathrm{n}=15$ ), defining 4 types of granulomas attributable to visceral Linguatulosis, according to the presence and features of cells at the centre of the lesion, the presence of presumably degenerated nymphal structures, location of inflammatory infiltrate, as well as location and amount of surrounding fibrous tissue. These lesions can concede a presumptive microscopic diagnosis. Also, different microscopic features of granulomas compatible with Linguatulosis analysed in this study suggest a temporal evolution of the lesions. The description of lesions generates a better understanding of the host-parasite interaction of this agent which has importance in both animal productivity and public health.

Key words: linguatulosis, microscopic diagnosis.
\end{abstract}

\section{INTRODUCTION}

Linguatulosis is a disease caused by the pentastomid parasite Linguatula serrata. This disease has a zoonotic character, therefore, it represents a risk to public health and produces important economic losses in livestock production (Taylor et al 2016).

L. serrata mainly infests mammals including humans and has an indirect life cycle in which the definite host are domestic or wild carnivores, while the intermediate host are herbivores, especially ruminants. The disease has two presentations: visceral and nasopharyngeal. The visceral form is presented by the intermediate hosts while the nasopharyngeal form is developed by the definitive host. Humans are considered an accidental host able to develop both forms of the disease (Machado et al 2006, Gunn and Pitt 2012). The larva hatches in the intestine and migrates to the liver and mesenteric, hepatic, and mediastinal lymph nodes, which are the target organs of the parasite. At this point the larva becomes encysted and after several moults, reaches its infective form called nymph (Azizi et al 2015).

Received: 06.06.2019.

Accepted: 08.10.2019.

${ }^{a}$ Escuela de Medicina Veterinaria, Facultad de Recursos Naturales y Medicina Veterinaria, Universidad Santo Tomás, Talca, Chile.

${ }^{b}$ Escuela de Medicina Veterinaria, Facultad de Recursos Naturales y Medicina Veterinaria, Universidad Santo Tomás, Concepción, Chile.

${ }^{c}$ California Animal Health and Food Safety Laboratory, San Bernardino, USA.

*Corresponding author: P Morales Muñoz; pmoralesm@ santotomas.cl
The diagnosis of visceral Linguatulosis is performed by visual identification of macroscopically compatible lesions and the detection of the nymph. Thus, the main affected organs are liver and mesenteric lymph nodes (Yakhchali and Tehrani 2013, Azizi et al 2015, Hajipour and Tavassoli 2019). The macroscopic lesions compatible correspond to well delimited, flat or slightly elevated, usually solitary greyish-white nodules of 2 to $4 \mathrm{~mm}$ diameter, located in the subcapsular area of the liver. Due to this feature and small size they can often go unnoticed, therefore the frequency of the diagnosis in animals could be underestimated (Valenzuela et al 1995, Castro et al 2015, Carrillo et al 2017).

The nymph of $L$. serrata is characterised by the possession of a saw-like cuticle with multiple transverse grooves, a ventral buccal apparatus with two pairs of peribuccal hooks and acidophilic glands, a complete digestive system and primordial genital organs. Moreover, in degraded nymphs, the hooks and remnants of the cuticle are often the only findings that allow the diagnosis of the infestation (Tappe and Büttner 2009, Rabeeh et al 2017).

In animals, few studies describe and characterise the inflammatory reaction triggered by L. serrata (Farjanikish and Shokrani 2016, Paredes and Muñoz 2016, Rabeeh et al 2017). In contrast, microscopic patterns of tissue reactions for each type of lesion have been described for humans, concerning the intensity of the inflammatory response, cell populations and the repair process of the surrounding tissue (Tappe et al 2006). This type of studies has allowed describing three types of diagnoses: etiopathological, sub-etiopathological and presumptive (Ma et al 2002).

The etiopathological diagnosis is based on the stereomicroscopic visualization of the intact nymph surrounded 
by a capsule with little or no adjacent cellular infiltration (Tappe and Büttner 2009). The sub-etiopathological diagnosis is conferred by the presence of at least one characteristic structure of the pentastomids or parts of the nymph within a macroscopically and microscopically compatible lesion (Tappe et al 2006). Finally, the presumptive diagnosis is based on the description of macroscopic or microscopic compatible lesions that could have been produced by $L$. serrata or another agent (Ma et al 2002).

Hajipour et al (2019) reported that L. serrata infestation in the mesenteric lymph nodes of the goats showed that the rate and intensity of infestation were greater in lymph nodes with altered colour and consistency. It was also revealed that the infestation rate was age-dependent, showing that the infestation increases as goats grow older. Moreover, the prevalence of infestation rate in female goats was significantly higher than that of male goats. The prevalence differs among animals depending on the location, probably climatic factors affect the survival of the eggs, temperature and humidity can play important roles in the epidemiology. The prevalence of $L$. serrata is higher in goats in comparison to other animals in Iran, which can be an important risk factor for human infestation (Hajipour and Tavassoli 2019).

Studies of the histologic lesions caused by L. serrata in animals would represent a contribution to improve the current understanding of parasite-derived pathogenic mechanisms, therefore, the objective of this study is to microscopically characterise the lesions produced by the parasite in the liver of cattle obtained from a slaughterhouse in Curico, Chile.

\section{MATERIAL AND METHODS}

In the city of Curicó, Chile, 269 bovine livers collected at a slaughterhouse were examined. The liver capsule was inspected, and several cuts were made in the visceral side of the organ. The analysis was carried between June and August 2018. The age and gender of each analysed animal were registered.

The number of animals was determined with a formula for finite populations (Aguilar-Barojas, 2005), where an $\mathrm{N}$ of 1,600 animals slaughtered monthly was considered, with a confidence of $95 \%$ and a precision of 0.05 .

For the presumptive macroscopic diagnosis of visceral Linguatulosis, the liver capsule was inspected, and 15 longitudinal cuts were made in search of compatible lesions (Valenzuela et al 1995, Castro et al 2015, Carrillo et al 2017). The detected compatible lesions were extracted with a margin of surrounding normal hepatic tissue of approximately $0.5 \mathrm{~cm}$, immersed in $10 \%$ formalin (v/v), labelled and sent to the laboratory for routine histological processing.

After 24 hours of fixation, all compatible lesions were cross-sectioned and analysed with a light stereomicroscope (Kyoto model Optical XTJ-4400) for the recognition of nymphs of $L$. serrata. The differential morphological characteristics described by Hamid et al (2012) were considered.

Samples with compatible lesions were subjected to routine histological processing and staining with Hematoxylin-Eosin (H-E). Subsequently, the slides were observed with a light optical microscope (Olympus CX22). A descriptive comparison was made with the presence and absence of nymphs, as well as the presence and predominance of inflammatory cell population based on some parameters of microscopic features of lesions proposed by Gibson-Corley (2013). Accordingly, lesions without nymphs, attributable to Linguatulosis, were classified as pentastomid granulomas type 1, 2, 3, and 4 according to the presence and features of the cells at the centre of the lesion, the presence of parasitic structures, the location of the inflammatory infiltrate, and the amount of the surrounding fibrous tissue.

\section{STATISTICAL ANALYSIS}

The present study corresponds to an observational and descriptive investigation.

\section{RESULTS AND DISCUSSION}

From the 269 livers examined, 18 (6.6\%) showed compatible macroscopic lesions and, therefore, a presumptive diagnosis of Linguatulosis. No nymphs were identified in any cross-section of the lesion with a light stereomicroscope. However, in 3 out of the 18 lesions, the whole nymph or fragments were histologically identified, establishing an etiopathological diagnosis of Linguatulosis. A fine continuous capsule was observed in one of the cases and an apparently intact nymph was visualised in its central content (figure 1). The infiltrate was composed predominantly of eosinophils, accompanied by lymphocytes and some macrophages. In two cases, a well-defined inflammatory infiltrate was observed, in close contact with nymphal structures such as spicules and hooks, surrounded by the remains of a thin discontinuous capsule (figure 1). The predominant inflammatory infiltrate was composed of lymphocytes and plasma cells together with some macrophages.

In Talca, Chile, Carrillo et al (2017) analysed bovine liver obtained at a slaughterhouse and described the macroscopic lesions compatible with Linguatulosis, which were afterwards etiologically confirmed by stereoscopy, obtaining a prevalence of $13.8 \%$ and $6,7 \%$ respectively. Castro et al (2015) indicated a $5.97 \%$ of prevalence in bovine lymph nodes. The diagnostic method of Linguatulosis in slaughterhouses relies on the macroscopic visualisation of compatible lesions with occasional identification of nymphs. Is important to consider the low size of the lesion and nymphs, this infection is hard to detect during the routine examination, especially when this is not a mandatory report disease (Ministerio de Salud 2002, Carrillo et al 2017, Hajipour and Tavassoli 2019). 


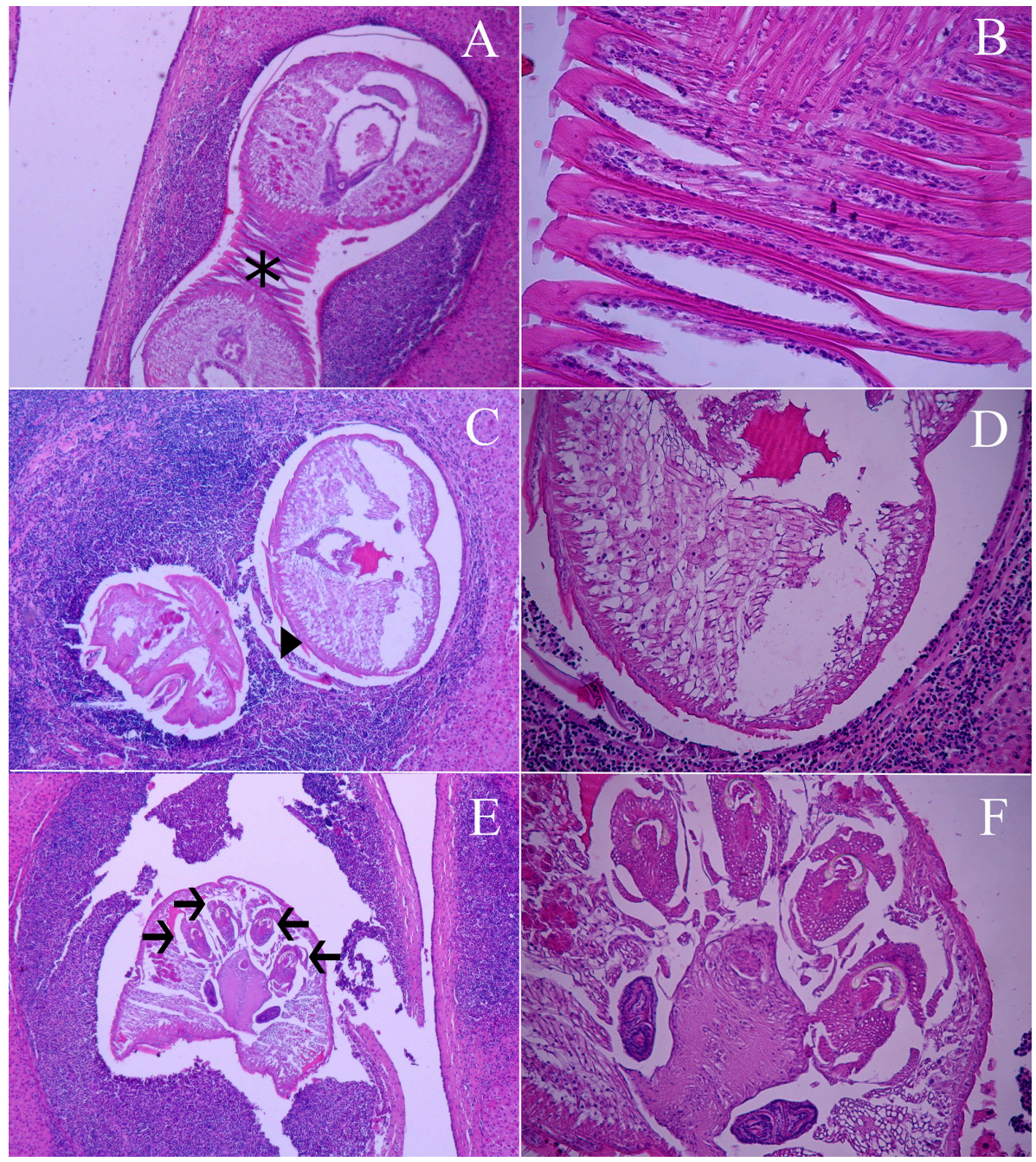

Figure 1. Pentastomid cyst in liver compatible with L. serrata, Hematoxylin-Eosin stain. A. longitudinal section of a nymph (10X). B. Transversal grooves detail (asterisk) (40X). C. Transversal section of a nymph with a peripheral inflammatory infiltrate (10X), the arrow indicates the spicules. D. Spicules (arrowhead) (40X). E. Transversal section of nymph head with peripheral inflammatory infiltrate (10X). F. Peribuccal hooks and acidophilic glands (arrows) (40X).

The lesions without the presence of nymphs $(n=15)$ were described and classified in 4 groups of pentastomid granuloma. Pentastomid Granuloma Type 1 (figure 2) $(\mathrm{n}=1 ; 8.33 \%)$ were formed by a dense, predominantly eosinophilic inflammatory core, accompanied by lymphocytes and macrophages, all circumscribed by a thin band of connective tissue and fibroblasts. In the case of Pentastomid Granuloma Type 2 (figure 2) $(n=7 ; 58.33 \%)$, they presented a cavity in the middle of the lesion with extensive loss of tissue. The inflammatory infiltrate was arranged in concentric layers of macrophages, followed by lymphocytes and lesser eosinophils, all circumscribed by a band of fibroblasts and fibrous tissue. Pentastomid Granuloma Type 3 (figure 2) (n=3;25\%) were characterised by tissue loss and a centre surrounded by concentric bands of inflammatory infiltrate constituted in centrifugal order by macrophages and lymphocytes interspersed with fibrous connective tissue and fibroblasts. Occasional foci of mineralisation were randomly identified. Finally, Pentastomid Granuloma Type 4 (figure 2) $(n=1,8.33 \%)$ presented a mineralised necrotic centre, surrounded by an inflammatory infiltrate composed of lymphocytes, macrophages and fibroblasts in similar proportions surrounded by areas of dystrophic mineralisation.

These results are similar to those described in humans, which have been used as a reference to make presumptive diagnoses attributable to L. serrata (Ma et al 2002). However, unlike those observed in human studies, sometimes the granulomas of the present study had a thin eosinophilic, translucent, layered, and acellular structure at its centre compatible with the chitin cuticle surrounding a nymph (figure 1). This structure could appear sectioned, folded or completely covering the interior of the lesion, in intimate contact with macrophages or cellular debris. In such 


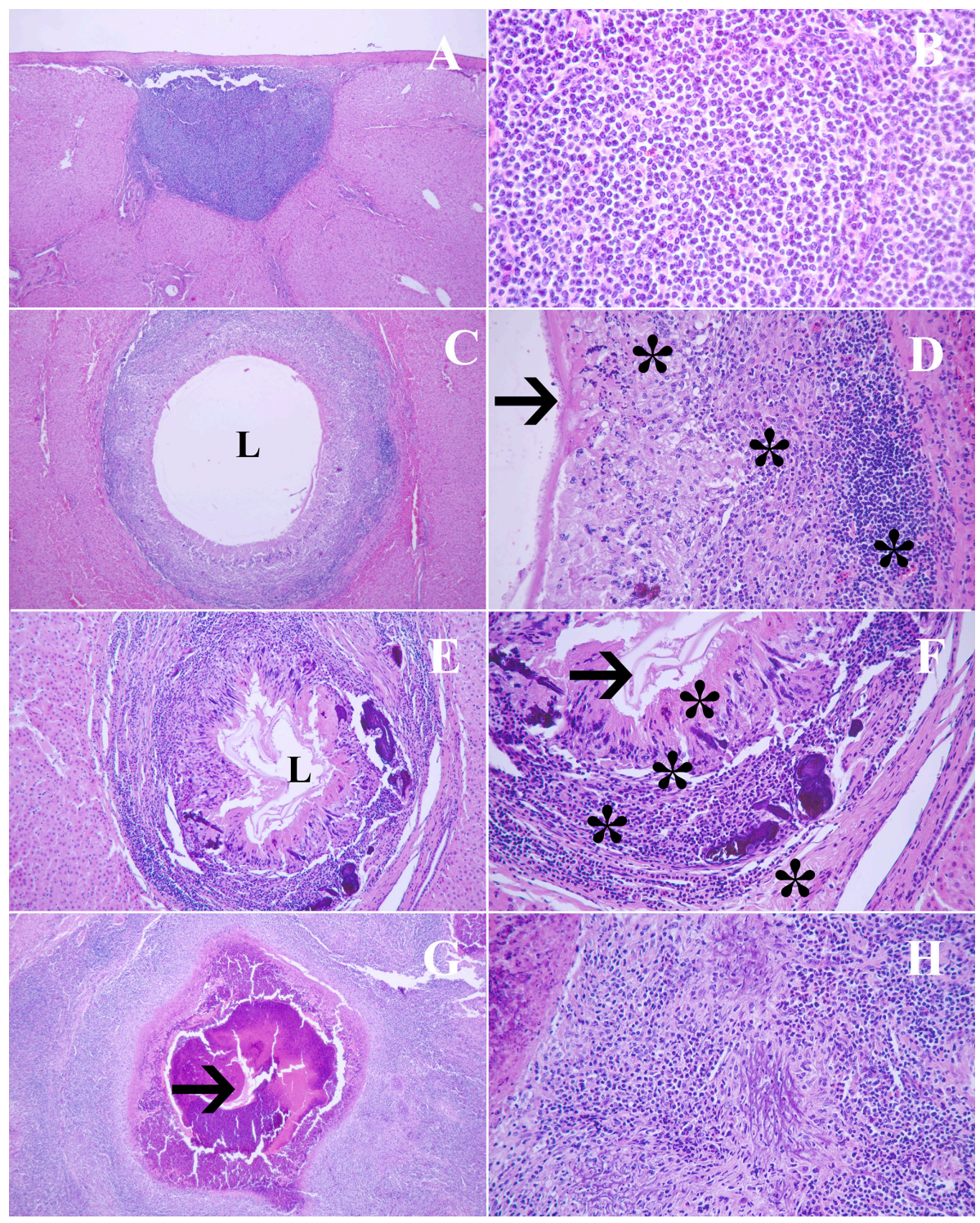

Figure 2. Pentastomid gramulomas in liver, Hematoxylin-eosin stain. A. Subcapsular granuloma type 1 (10X). B. Mixed inflammatory infiltrate with predominantly eosinophilic cells (40X). C. Granuloma type 2 with central lumen (L) (40X). D. Eosinophilic connective tissue around the lumen (arrow) and mixed inflammatory cellular infiltrate (asterisks). E. Granuloma type 3 with irregular lumen and foci of mineralization (10X). F. Eosinophilic structure intraluminal compatible with pentastomid cuticle (arrow), connective tissue (arrowhead) and different inflammatory cellular infiltrates (asterisks). G. Granuloma type 4 with a necrotic and mineralized centre and abundant peripheral inflammatory infiltrate (10X). H. Mixed inflammatory infiltrate around the necrotic tissue (40X).

cases, the pentastomid granulomas would be delivering a sub-etiopathological diagnosis (Tappe and Büttner 2009). In the case of Pentastomid Granuloma Type 1 $(\mathrm{n}=1)$, this was composed of a predominantly eosinophilic inflammatory infiltrate, similar to that observed in lesions with the presence of a nymph and an integral capsule; but no nymphal structures were visualised. Rabeeh et al (2017) describe fibrosis of the infected lymph nodes around the parasite in different animals, in some cases indicating a granulomatous reaction formed by giant cells, macrophages, lymphocytes, and eosinophils around the degenerated nymphs.
In this study, it is proposed that all lesions correspond to the different stages and modifications suffered by the nymph in their natural course of degradation and reactions with the surrounding tissue. We propose that the lesion progresses with a nymph with a viable capsule that induces an early eosinophilic response, passing through the progressive capsular and cuticular rupture with almost complete parasitic destruction which leaves a cavity, with parallel recruitment of large numbers of macrophages and lymphocytes around the parasite jointly with the formation of a granuloma similar to the foreign body reaction type. The latter is characterised by the deposit of connective 
tissue showing a contraction of the lesion with folding or fragmentation of the internal structures, leading to the filling of the cavity left by the nymph with inflammatory cellular debris and mineralisation.

Regarding the few histopathological descriptive reports existing in animals, further contributions with more detailed characterisations of the lesions induced by $L$. serrata are needed to reach a more consistent presumptive diagnosis. Moreover, recognising and describing the predominant nature of inflammatory responses in lesions could generate a basis for further discussion and a better understanding of the host-parasite interaction of this agent which has importance in both animal productivity and public health.

\section{REFERENCES}

Aguilar-Barojas S. 2005. Fórmulas para el cálculo de la muestra en investigaciones de salud. Vol.11. Secretaría de Salud del Estado de Tabasco, México, Pp 333-338.

Azizi H, Nourani H, Moradi A. 2015. Infestation and pathological lesions of some lymph nodes induced by Linguatula serrata nymphs in sheep slaughtered in Shahrekord Area (Southwest Iran). Asian Pac J Trop Biomed 7, 574-578.

Carrillo M, Morales P, Carvallo F, Abarca C. 2017. Prevalencia de linguatulosis hepática en bovinos beneficiados en una planta faenadora de carnes, Talca, Chile. Rev Investig Vet Perú 28, 169-177.

Castro J, Naupay A, Fajardo N, Trevejo G, Almeyda V, et al. 2015. Prevalencia de ninfas de Linguatula serrata en ganado bovino en camales de Lima, Perú. Rev Investig Vet Perú 26, 310-316.

Farjanikish G, Shokrani H. 2016. Prevalence and morphopathological characteristics of linguatulosis in one-humped camel (Camelus dromedarius) in Yazd, Iran. J Parasitol Res 115, 3163-3167.

Gibson-Corley K, Olivier A, Meyerholz D. 2013. Principles for valid histopathologic scoring in research. Vet Pathol 50, 1007-1015.

Gunn A, Pitt SJ. 2012. Arthropod parasites. In: Parasitology: An Integrated Approach. Wiley Blackwell, West Sussex, UK, Pp 151-153.
Hajipour N, Soltani M, Mirshekar F. 2019. Effect of age, sex, and season on the prevalence of Linguatula serrata infestation in mesenteric lymph nodes of goats slaughtered in Tabriz, Iran. Trop Anim Health Prod 51, 879-885.

Hajipour N, Tavassoli M. 2019. Prevalence and associate factors of Linguatula serrata infection in definitive and intermediate hosts in Iran and other countries: A systematic review. Veterinary Parasitology: Regional Studies and Reports 16,100288.

Hamid T, Hossein YD, Mehran BB, Masood F, Hamid E. 2012. A case report of Linguatula serrata infestation from rural area of Isfahan City, Iran. Adv Biomed Res 1, 42.

Ma K, Qiu M, Rong Y. 2002. Pathological differentiation of suspected cases of pentastomiasis in China. Trop Med Int Health 7, 166-177.

Machado MA, Makdissi FF, Canedo LF, Martino RB, Crescentini F, et al. 2006. Unusual case of pentastomiasis mimicking liver tumor. J Gastroenterol Hepatol 21, 1218-1220.

Ministerio de Salud. 2002. Norma general técnica sobre inspección médico veterinaria de las reses de abasto y de sus carnes y criterios para la calificación de aptitud para el consumo humano. Ministerio de Salud, Santiago, Chile.

Paredes E, Muñoz M. 2016. Descripción histopatológica de ninfas de Linguatula serrata en hígados de bovinos faenados en Valdivia, Chile. Rev Med Vet 9, 73-81.

Tabaripour R, Fakhar M, Alizadeh A, Reza Youssefi M, Tabaripour R, et al. 2017. Prevalence and histopathological characteristics of Linguatula serrata infection among slaughtered rumiants in Mazandaran Province, northern Iran. Comp Clin Pathol 26, 1259-1265.

Tappe D, Winzer R, Bütnner D, et al. 2006. Linguatulosis in Germany. Emerg Infect Dis 126, 1034-1036.

Tappe D, Büttner D. 2009. Diagnosis of human visceral pentastomiasis. PLoS Negl Trop Dis 3, 320.

Taylor M, Coop R, Wall R. 2016. Veterinary Parasitology. $4^{\text {th }}$ ed. Wiley Blackwell, West Sussex, UK, Pp 257-258.

Valenzuela G, Bascuñan M, Bayer L, Ernst S. 1995. L. serrata infections in bovine livers in Valdivia, Chile. Arch Med Vet 27, 29-34.

Yakhchali M, Tehrani AA. 2013. Histopathological changes caused by the nymph stage of Linguatula serrata in the mesenteric lymph nodes of goats. Acta Vet Hung 61, 36-41. 
